Questions vives

\section{Questions Vives}

Recherches en éducation

$N^{\circ} 25$ | 2016

L'activité des enseignants face au décrochage scolaire : Quelles difficultés et quelles mises en œuvre professionnelles?

\title{
Intervenir pour aider les enseignants dans la prise en charge des élèves décrocheurs
}

\author{
An intervention to help teachers support student dropouts
}

\section{Sylvie Moussay et Véronique Blanjoie}

\section{(2) OpenEdition}

Édition électronique

URL : http://journals.openedition.org/questionsvives/1887

DOI : 10.4000 /questionsvives. 1887

ISSN : $1775-433 \mathrm{X}$

\section{Éditeur}

Université Aix-Marseille (AMU)

\section{Édition imprimée}

Date de publication : 7 juillet 2016

ISBN : 978-2-912643-49-0

ISSN : 1635-4079

Référence électronique

Sylvie Moussay et Véronique Blanjoie, «Intervenir pour aider les enseignants dans la prise en charge des élèves décrocheurs », Questions Vives [En ligne], № 25 | 2016, mis en ligne le 05 septembre 2016, consulté le 20 avril 2019. URL : http://journals.openedition.org/questionsvives/1887 ; DOI : 10.4000/ questionsvives.1887

Ce document a été généré automatiquement le 20 avril 2019.

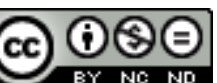

Questions Vives est mis à disposition selon les termes de la licence Creative Commons Attribution Pas d'Utilisation Commerciale - Pas de Modification 4.0 International. 


\title{
Intervenir pour aider les enseignants dans la prise en charge des élèves décrocheurs
}

\author{
An intervention to help teachers support student dropouts
}

\author{
Sylvie Moussay et Véronique Blanjoie
}

\section{Introduction}

1 Confrontés à une succession d'injonctions au changement pour lutter contre le décrochage scolaire, les enseignants expriment souvent leur impuissance à innover pour aider les élèves décrocheurs. Ce désarroi s'accompagne d'une forte démotivation au travail, d'un "inconfort intérieur» pouvant se traduire par un découragement que certains définissent comme un épuisement professionnel face à la complexité du travail. C'est particulièrement dans ce contexte que le processus de décrochage professionnel fait l'objet de questionnement et d'investigation (Flavier \& Moussay, 2014). Selon certaines études, le décrochage enseignant est défini comme l'abandon de la profession désignant ainsi une rupture professionnelle (Mukamurera \& Bouthiette, 2008). Les jeunes enseignants, particulièrement confrontés à de multiples défis et à des dilemmes professionnels (Ria, 2015) quittent la profession enseignante au cours des premières années d'enseignement (OCDE, 2005; Skaalvik \& Skaalvik, 2011). Dans ce cas, le décrochage professionnel désigne le départ prématuré de la profession enseignante (Karsenti, Collin \& Dumouchel, 2013). Ainsi, aux États-Unis, « $11 \%$ quittent la profession à l'issue de leur première année d'exercice, et $39 \%$ dans les cinq ans » (Borman \& Dowling, 2008). De la même façon, en Belgique francophone, « quatre sur dix enseignants du Secondaire ont abandonné au cours de leurs cinq premières années » (Lothaire, Dumay \& Dupriez, 2012). Cette problématique du décrochage des enseignants dévoile les phénomènes de fuite, de déperdition (Blanjoie, 2014), de turnover et d'attrition (MacDonald, 1999; Karsenti \& 
Collin, 2009) et l'ampleur des épreuves subjectives traversées qui décident souvent de leur engagement - ou dégagement professionnel (Barrère, 2002).

\section{Problématique de l'étude}

2 Dans nos travaux de recherche, nous nous intéressons à une forme particulière de décrochage des enseignants dont l'origine provient d'une amputation du pouvoir d'agir (Clot, 2008) sur les situations de travail, révélant ainsi une rupture avec le milieu de travail et une impuissance à poursuivre l'activité professionnelle avec des élèves décrocheurs c'est-à-dire des élèves refusant de s'engager dans le travail scolaire (Méard, 2014). En référence aux travaux en psychopathologie du travail et en clinique de l'activité, le décrochage professionnel pourrait être associé aux rapports entre l'activité professionnelle et l'atteinte de la santé au travail. L'impossibilité de poursuivre le travail avec les élèves décrocheurs, et de s'appuyer sur un « répondant collectif » pour échanger sur les manières possibles de réguler le décrochage scolaire fragilise l'activité individuelle $\mathrm{du}$ professionnel. Les enseignants éprouvent ainsi une grande solitude face au désengagement des élèves dans la tâche scolaire. Cette situation de travail peut dans certains cas devenir insoutenable (Clot \& Gollac, 2014). En d'autres termes, l'absence d'engagement des élèves dans la tâche, lié au déficit de sens qu'ils accordent au travail scolaire affecte le sens que donne l'enseignant à son activité (Méard, 2014) et crée en conséquence des situations de travail contrarié (Clot, 2010). Ces considérations invitent à envisager le décrochage professionnel et le décrochage scolaire comme des processus interpénétrés. Dans ce contexte, nos interventions consistent à collaborer avec les professionnels et les élèves pour les aider à construire un nouveau sens aux situations de travail dans lesquelles ils sont conjointement engagés (Flavier \& Moussay, 2014). Inspirées par la démarche du Change Lab (Engeström, 2001 ; Engeström \& Sannino, 2013 ; Virkkunen \& Newnham, 2013) mais ancrées essentiellement dans une méthodologie engageant les professionnels dans une nouvelle expérience (Clot, 2008), nos interventions visent le développement de l'activité de travail par le biais d'analyses et d'échanges professionnels susceptibles d'aider les enseignants à dépasser les situations d'enseignement problématiques. Chercheurs et participants sont ainsi impliqués dans une approche centrée sur l'activité et dans un processus collaboratif de conception et de mise en œuvre des innovations pratiques.

Dans le cadre de cet article, notre contribution présente les circonstances dans lesquelles les enseignants du collège s'engagent dans l'espace d'analyse du travail pour prendre en charge collectivement la problématique du décrochage scolaire. La première partie situe le fondement épistémologique de notre intervention en référence aux théories de l'activité. La seconde partie présente la méthodologie, notamment le protocole de l'intervention invitant les professionnels à analyser seuls ou à plusieurs leur activité professionnelle pour identifier les tensions au cœur du décrochage. La troisième partie sur les résultats est structurée autour des différentes étapes d'analyse du travail considérées comme autant d'occasions de développer l'activité. Enfin, la discussion puis la conclusion permettent de revenir sur l'espace d'analyse du travail en termes de ressources pour faire face au décrochage des élèves et de dynamique collective autour d'un partage des expériences du travail. 


\section{Cadre théorique}

\subsection{Une intervention développementale}

Dans une perspective développementale, la pratique de l'intervention dans et sur les milieux de travail s'engage dans une voie désignée comme une recherche fondamentale de terrain se situant au-delà d'une démarche explicative qui dissout son objet et d'une démarche compréhensive qui le rejoint trop vite (Clot, 2008). L'intervention comme action ou pratique vise la mise en place d'un cadre d'échanges professionnels favorisant l'analyse de l'activité dans toute sa dynamique et sa complexité, l'objectif étant d'identifier les éléments de cette complexité, notamment ceux qui sont à l'origine de contradictions au cœur de l'activité professionnelle. L'intervention est ainsi orientée explicitement vers le dépassement des tensions et des dilemmes vécus dans l'activité et la résolution de problèmes réels.

\subsection{Le principe de la stimulation duale}

$5 \quad$ Le présupposé théorique selon lequel le sujet se développe par la médiation des outils peut être étayé par le principe épistémologique de la stimulation duale (Vygotski, 1934/1997). Ce principe fait référence au processus par lequel le sujet peut intentionnellement dépasser une situation conflictuelle et modifier une situation ou résoudre des problèmes (Sannino, 2012; Engeström \& Sannino, 2013). Dans notre étude, ce processus de stimulation duale s'appuie sur l'usage d'entretiens d'autoconfrontation et la mise en place de confrontations collectives dont l'objectif est de provoquer et susciter la construction de nouvelles significations sur l'activité. Les extraits vidéo lors des entretiens d'autoconfrontation ainsi que les commentaires et réactions des collègues au cours des confrontations collectives sont envisagés comme autant de stimuli et de moyens auxiliaires au service du développement de l'activité c'est-à-dire du renouvellement des actions et des motifs d'action (Leontiev, 1981). Notre intervention met ainsi à disposition des outils que les professionnels s'approprient pour interroger l'activité réalisée, notamment les situations de travail contrariées avec les élèves décrocheurs et envisager de nouveaux buts d'action associés à des motifs différents de ceux initialement suivis.

\subsection{Le développement du pouvoir d'agir du collectif professionnel}

6 L'intervention s'appuie sur le postulat d'une conception des sujets comme acteurs du changement et comme principaux artisans ayant l'intention de transformer leurs pratiques. C'est précisément l'intervention développementale (Clot \& Kostulski, 2011) qui contribue à favoriser la participation et l'engagement des professionnels dans une collaboration. L'un des principaux enjeux réside ainsi dans le dépassement de la juxtaposition d'actions et de problématiques professionnelles individuelles pour tendre vers la constitution d'un collectif (Clot, 2008) capable de transformer l'expérience vécue en moyen d'en vivre une autre (Vygotski, 1934/1997). Cette perspective suppose que l'intervention favorise le développement d'un intérêt collectif pour reprendre en main, analyser et transformer les pratiques pédagogiques. Le défi de l'intervention est alors de développer une intention, une raison effective pour une participation dans l'analyse des 
problèmes plutôt que de rechercher des solutions immédiates aux problèmes (Virkkunen, 2006).

\section{Méthodologie}

\subsection{Contexte et participants}

7 L'intervention ${ }^{1}$ s'est déroulée dans un collège classé en réseau d'éducation prioritaire situé dans la banlieue lyonnaise. Lors d'un premier contact avec la principale de l'établissement à la rentrée 2014, les enseignants et d'autres professionnels (assistants pédagogiques, professeurs stagiaires, conseiller principal d'éducation...) ont exprimé leur difficulté à gérer en classe les élèves en situation de décrochage scolaire. Parallèlement, ils ont évoqué dans un questionnaire ${ }^{2}$ leur intérêt de mieux comprendre l'activité en classe et d'avoir une meilleure visibilité du travail réalisé avec ces élèves. Avec l'accord de la principale du collège, nous avons proposé de mettre en place un espace d'analyse et de partage des expériences du travail, permettant aux professionnels d'interroger leur pratique avec les élèves décrocheurs. Dès le mois de septembre, quinze enseignants volontaires se sont engagés dans cet espace d'analyse et quatre enseignants supplémentaires les ont rejoints en février.

\subsection{Protocole de l'intervention}

Les temps d'analyse du travail ont eu lieu sur les heures de concertation et de formation ${ }^{3}$ du réseau de l'éducation prioritaire. Cette inscription dans l'emploi du temps des enseignants du collège $\mathrm{T}$ a été un élément déterminant et favorable à l'engagement des professionnels. L'intervention est structurée autour de quatre étapes : la première étape consiste à questionner la pratique existante afin d'identifier les contradictions et les problématiques professionnelles avec les élèves décrocheurs ; lors de la seconde étape, les professionnels se filment en classe puis choisissent deux ou trois extraits vidéo en lien avec les problématiques précédemment identifiées. Ces extraits vidéos de l'activité en classe d'une durée comprise entre une à six minutes deviennent les supports des entretiens d'autoconfrontations simples et croisées. Au cours de la troisième étape, les analyses produites sont portées à la discussion des professionnels. Enfin la dernière étape invite les professionnels à expérimenter les nouvelles modalités de travail dont les principes organisateurs ont fait l'objet d'un échange, voire d'un consensus professionnel. Ces différentes étapes ont été pilotées par la chercheuse et deux enseignantes coordonnatrices dont le rôle était de faciliter les analyses, les confrontations et les échanges exploratoires sur l'activité en classe. Précisons que les deux coordonnatrices ont suivi plusieurs séquences de formation à l'analyse du travail.

\subsection{Recueil et analyse des données}

9 En référence à l'approche développementale de l'intervention, le recueil et l'analyse des données ont été réalisés par cycles successifs. Des notes d'observation, des données d'entretiens d'autoconfrontation, et celles des discussions en plénière ont été recueillies. L'analyse a consisté à transcrire verbatim l'ensemble des données issues des quatre étapes, l'objectif étant dans un premier temps de pouvoir rendre compte du processus : (i) le 
questionnement des problématiques existantes; (ii) les analyses de l'activité lors des entretiens d'autoconfrontation; (iii) les confrontations ouvertes sur la conception d'une nouvelle forme de pratique ; (iv) l'analyse collective, après expérimentation, du potentiel et des limites de cette nouvelle forme de pratique en vue de sa consolidation. Dans un second temps, en référence au cadre conceptuel des théories de l'activité, il s'est agi de relever dans le corpus l'expression des tensions propres aux actions et aux motifs concurrents énoncés par les professionnels, ainsi que les arbitrages effectués entre différentes actions pour résoudre les situations d'enseignement problématiques devant les élèves décrocheurs.

\section{Résultats}

10 Quatre résultats permettent de documenter la manière dont les professionnels du collège se sont impliqués dans le nouvel espace d'analyse du travail pour questionner les situations de décrochage scolaire et développer de nouvelles modalités d'actions.

\subsection{La description historique des pratiques pour identifier les contradictions (étape1)}

11 Lors des premières réunions, nous avons examiné avec les enseignants la problématique du décrochage scolaire, notamment les évolutions institutionnelles (réformes sur la lutte contre l'échec scolaire) ainsi que les différentes formes de dispositifs mis en place dans leur collège pour aider les élèves décrocheurs. Lors des échanges, il a été rapidement identifié par le collectif les contradictions suivantes. La première contradiction concernait le « grand fossé entre deux mondes » comme l'ont exprimé les enseignants entre les dispositifs de lutte contre le décrochage et l'activité en classe.

\section{Entretien avec Naïma, enseignante de français (septembre 2014)}

$\mathrm{N}$ : On a vraiment l'impression de faire fonctionner deux mondes différents; comment les élèves peuvent s'y retrouver si on ne prend pas en compte dans nos classes ce que nos élèves font dans ces dispositifs alors qu'on pensait les avoir un peu raccrochés aux activités scolaires et hop brutalement quand ils viennent en classe ils décrochent à nouveau et ça ça s'explique par l'absence évidente de coordination entre la classe et les dispositifs d'aide.

\section{Entretien avec Paul, enseignant Histoire-Géographie (septembre 2014)}

P : Parmi les éléments du contexte il faut quand même pas oublier la pression depuis quelques années la pression sur une plus grande efficacité des dispositifs de soutien et cette visée des programmes on n'a pas forcément trouvé le cadre ou la bonne liaison entre les dispositifs et ce qu'on fait en classe [...]donc en fait je me pose vraiment la question de l'efficacité de tous ces dispositifs qui vont qui viennent chaque année [...] je pense qu'on pourrait envisager toutes ces aides à l'intérieur de la classe [...] mettre en place des dispositifs de soutien mais pas en dehors plutôt dans la classe.

La deuxième contradiction s'expliquait selon les enseignants par «le frein d'une forte tradition». 


\section{Entretien avec Sarah, enseignante de Français (octobre 2014)}

S: Il a toujours existé ici mais je crois que c'est le cas dans les autres établissements ; ici entre les profs il y a une certaine distance et méfiance, c'est une forte tradition qui nous empoisonne; au plan des pratiques, il en résulte une certaine incompréhension mutuelle quant aux activités qu'on fait avec les élèves [... ] le pire c'est d'avoir les mêmes élèves, en fait on a très peu d'informations sur leur comportement dans les autres cours parce qu'on n'a jamais le temps d'en parler entre nous.

13 La troisième contradiction a émergé d'une discussion sur un dispositif impulsé par la principale du collège en matière de lutte contre le décrochage scolaire : «les entretiens individualisés » avec les élèves décrocheurs. En discutant de ce dispositif, les enseignants ont évoqué un dispositif mal conçu aboutissant, souvent par manque de temps, à convoquer les élèves sur les heures de cours. Le témoignage de deux enseignants impliqués dans la mise en œuvre de ce dispositif a incité les autres professionnels à analyser la situation de manière plus globale en mettant en avant les éléments organisationnels qui contraignaient les entretiens individualisés avec les élèves décrocheurs.

14 Ces premiers échanges ont permis de dresser quelques constats: un décrochage au collège situé à l'intersection de différentes activités (les activités en classe et les dispositifs d'accompagnement personnalisé), associé à des problèmes d'organisation du travail et de coopération professionnelle. Nous avons noté également chez les enseignants une grande lassitude illustrée par exemple par les propos de Fabienne, enseignante de Sciences de la Vie et de la Terre « on a beau revoir nos méthodes, nos cours et les projets d'aide, on a beau s'impliquer dans des projets toujours plus nombreux, le résultat est là, des élèves qui nous échappent et qui refusent de se mettre en activité dans nos classes ».

\subsection{L'analyse de l'activité pour évoquer les tensions (étape 2)}

À partir des données empiriques (données d'enregistrement de l'activité en classe, données issues des travaux d'élèves et des traces écrites de la préparation de leçon), nous avons proposé aux professionnels de poursuivre l'analyse de l'activité des élèves décrocheurs à travers des entretiens d'autoconfrontation.

\section{Autoconfrontation avec Fabienne, enseignante de sciences de la vie et de la Terre (novembre 2014)}

F : c'est difficile pour moi de rentrer dans le vif du sujet alors que certains élèves sont à la traine ils n'ont pas encore sorti leurs cahiers, leurs livres [...] moi ça me décourage à chaque fois [...] je suis parfois à deux doigts de partir du cours ; mais c'est vrai que pour les élèves peu motivés qui sont là, on les aide pas trop [...] je pense au rituel, on n'a pas les mêmes rituels de début de cours donc les élèves sont un peu perdus et pour certains c'est le point de départ d'un décrochage.

Autoconfrontation avec Lilian, enseignant de technologie (novembre 2014)

$\mathrm{L}$ : Là j'ai donné les consignes et ensuite ils doivent aller vers un ordinateur j'essaye de développer le plus possible l'autonomie et ça fait plusieurs fois que cet élève arrivé au poste ne sait pas ouvrir sa session; il prend souvent un temps fou [...] là c'est typique il me demande de lui rappeler la consigne; en fait le challenge pour eux une fois que j'ai donné les consignes, c'est de les garder en mémoire pendant très longtemps jusqu'au geste d'ouverture de la session [...] avant je faisais 
autrement, j'écrivais au tableau « étape 1 , tu fais ça, étape 2, ça » mais en fait je me suis aperçu que ça cloisonnait l'élève dans ce qu'il pouvait faire et il passait son temps à déchiffrer ce que j'avais mis au tableau comme procédures à suivre ; donc après j'ai décidé de les laisser se débrouiller c'est ce que je fais là ; mais lui il est complètement perdu [...] en fait je l'ai perdu et je m'énerve ; je lui dis de s'asseoir et là je l'isole encore plus [...] mais tu vois ce gamin, je serai vraiment curieux de savoir comment il se comporte dans les autres cours, problème ou pas problème.

\section{Autoconfrontation croisée avec Floriane, enseignante de français et Juliette, enseignante d'histoire-géographie (novembre 2014)}

$\mathrm{J}$ : là c'est flagrant ils mettent un temps fou pour travailler mais c'est quand même intéressant de voir F. (élève décrocheur); sans M. (autre élève), j'imagine comme dans mon cours que $\mathrm{F}$ aurait déjà abandonné depuis longtemps.

$\mathrm{F}$ : mais je ne suis pas satisfaite de cette organisation

$\mathrm{J}$ : et pourtant, F. je le connais il aurait jamais bossé sans $\mathrm{M}$.

F : oui mais je vois quand même que les élèves à qui j'ai donné la responsabilité d'aider un autre élève ont du mal à savoir ce qu'il faut faire $\mathrm{J}$ : ah mais on pourrait, c'est sûr, guider un peu plus quand on leur demande d'aider un autre élève ; mais moi je constate que là, ça te permet quand même de te libérer et tu peux comme ça t'occuper de G. (autre élève décrocheur)

$\mathrm{F}$ : oui oui mais c'est pas tout à fait ce que j'attendais comme résultat je ne suis pas persuadée que F. soit en activité là ; il regarde la feuille du copain qui l'aide, recopie et mis à part ces occupations est-ce qu'il apprend quelque chose? L'avantage ici c'est qu'il n'ait pas renoncé comme il l'a déjà fait dans mes autres cours Chercheuse : et alors pourquoi tu n'es pas satisfaite?

F : c'est tellement complexe; je crois que la mise en activité de ces élèves qui sont vraiment prêts à renoncer suppose des conditions, un autre aménagement de la classe, une autre forme de travail, quand je vois que j'ai laissé à l'identique l'espace de la classe alors que l'aide entre élèves, j'y crois, pour éviter que certains décrochent mais je crois surtout que cela suppose une autre conception de la classe Chercheuse : tu veux dire une autre façon de faire classe?

$\mathrm{F}$ : oui déjà oui une autre façon de faire classe, mais aussi d'organiser l'espace et puis le travail avec ces élèves tuteurs ; et surtout je pense qu'il serait intéressant de savoir ce qu'il se passe vraiment dans cette doublette, lui qui d'habitude comme tu dis refuse de travailler.

Les enseignants ont ainsi décrit leur travail sous la forme de conflits vécus comme des situations problématiques qui viennent entraver les attentes professionnelles. Fabienne va jusqu'à définir son activité comme décourageante car difficile ou impossible à réaliser : "ça me décourage à chaque fois, je suis parfois à deux doigts de partir du cours». Elle a considéré l'absence de rituels communs à l'équipe enseignante à l'origine du décrochage des élèves; des rituels qui pourraient aider les élèves à se repérer, à construire des habitudes de travail favorisant une entrée plus rapide dans les apprentissages. Finalement, la question soulevée par Fabienne est celle de l'harmonisation de gestes professionnels susceptibles de faciliter l'entrée de l'élève dans la tâche scolaire. La problématique du décrochage des élèves est ainsi envisagée dans un cercle d'actions collectives et coopératives entre les enseignants. De son côté, Lilian a évoqué la tension entre des actions passées " avant je faisais autrement j'écrivais au tableau "étape 1 tu fais ça étape 2 ça" " et la décision de ne plus écrire au tableau les procédures pour inciter les élèves à mémoriser les étapes d'ouverture d'une session sur le poste informatique. Face aux images, il a pris conscience des limites de ses actions pour un élève en particulier «mais lui il est complètement perdu; en fait je l'ai perdu et je m'énerve [...] je lui dis de s'asseoir et là je l'isole encore plus ». Dans ce cas, l'analyse de l'activité a permis à l'enseignant d'exprimer 
les actions conduisant au décrochage d'un élève. Parallèlement à cette analyse, Lilian a perçu un intérêt professionnel à avoir davantage d'informations sur l'attitude de cet élève dans les autres cours.

Enfin, dans le dernier extrait d'entretien, les deux enseignantes ont questionné un dispositif mis en place pour favoriser la mise en activité des élèves. En commentant les images vidéo du cours de Floriane, Juliette a souligné l'intérêt du dispositif tutoral d'une part pour les élèves susceptibles d'abandonner " F. je le connais il aurait jamais bossé sans M. » et d'autre part pour l'enseignante «là ça te permet quand même de te libérer et tu peux comme ça t'occuper de G. ». Mais Floriane ne partage pas totalement cet avis; elle s'attarde davantage sur l'activité des élèves tuteurs "qui ne savent pas quoi faire » et sur celle d'un élève tutoré « je ne suis pas persuadée que F. soit en activité là ; il regarde la feuille du copain qui l'aide, recopie et mis à part ces occupations est-ce qu'il apprend quelque chose?». Plus loin, chacune leur tour, elles proposent de nouvelles actions « guider un peu plus quand on leur demande d'aider un autre élève » " aménager et organiser autrement l'espace de la classe ».

$\mathrm{Au}$ cours des entretiens, chaque enseignant a identifié des éléments générateurs du décrochage scolaire (absence de rituels communs; modalités d'intervention excluant l'élève) et souligné les limites de certaines mises en œuvre telles le tutorat entre élèves qui suppose d'autres actions (apprendre au tuteur-élève à conseiller) pour une meilleure efficacité en classe.

\subsection{La confrontation collective pour impulser de nouvelles réalisations (étape 3)}

19 Au cours d'une session de formation $\mathrm{REP}^{4}{ }^{4}$ au sein du collège, les professionnels sont invités à une reprise des traces vidéo de l'activité et des analyses produites lors de l'étape précédente. La confrontation collective a favorisé les débats sur le potentiel des dispositifs mis en place pour les élèves décrocheurs, ainsi que les analyses croisées entre le point de vue de la chercheuse et celui des enseignants.

20 L'extrait d'une confrontation collective illustre cette mise en débat. Juliette, enseignante d'Histoire-Géographie accepte de reparler du dispositif «îlots» mis en place dans une classe de $6^{e}$. Elle le décrit comme "assez mouvementé parce que pas encore stable», en expliquant que depuis son entrée au collège, elle a pris conscience que «les élèves qui décrochaient, c'était pas seulement à cause de lacunes disciplinaires mais plutôt à cause de l'absence d'apports méthodologiques». Juliette précise que pour elle "qu'il est devenu vital pour les élèves de travailler autrement ». Sa description fait émerger les stratégies d'aide au travail qu'elle n'a pas réussi à développer au cours des premières années. Tout en visionnant la séquence vidéo de son activité en classe, elle dit avoir eu recours au travail en îlots " parce qu'elle avait appris ici qu'il fallait s'occuper en priorité des élèves en décrochage alors que son projet visait également la prise en compte des quelques bons élèves du collège ». Elle poursuit en décrivant les buts et les opérations, «j'ai changé l'aménagement normal de la classe [...]2 ou 3 bureaux pour construire un îlot et là, j'ai 6 îlots dans la classe avec des niveaux différents à l'intérieur de chaque îlot[...]cet élève, Ahmed, il se débrouille je l'ai mis dans cet îlot, c'est en quelque sorte une ressource que je mets à disposition des autres qui ont du mal à s'impliquer dans le travail que je donne; le bénéfice de tout ça c'est que ça règle pas mal la gestion de l'hétérogénéité; moi je pense et pour la mise en activité, j'ai l'impression que ça les motive de travailler avec un autre élève ». La présentation de Juliette permet à chaque collègue de s'immerger dans une histoire professionnelle. Les discussions au sein du collectif sont 
ensuite l'occasion d'argumenter en faveur du dispositif : «là sur la vidéo, on voit des élèves courbés sur leur feuille, ils travaillent tous, pas de bruits parasites, c'est bien une mise en activité pour l'ensemble des élèves »; de revisiter une idée prise pour acquis : "j'ai peur que l'élève décrocheur qui se trouve avec le bon soit toujours dans l'attente "; d'apporter une nuance quant à l'impact du travail en îlots : «les élèves engagés dans ce dispositif se mettent au travail mais qu'apprennent-ils vraiment?»; de soulever de nouvelles questions sur les difficultés des élèves à apprendre dans ce dispositif: "mais là c'est Ahmed qui lui donne la phrase mais Daniel lui il l'écoute et il copie, est-ce qu'il a vraiment compris?»; d'identifier et d'expliciter de nouveaux potentiels pour faire évoluer le travail en îlots: "cette méthode elle suppose quand même qu'on travaille sur les consignes qu'on donne aux élèves dans les îlots"; de reconnaître les limites de cette méthode d'enseignement: «travailler comme ça, c'est du temps aussi, après on a des impératifs par rapport aux programmes, on peut pas utiliser cette méthode à tous les cours et la question est de savoir si un élève qui raccroche grâce au travail en îlot peut décrocher plus facilement lorsque le cours n'est plus organisé en îlots »; de questionner les implications d'une telle organisation pédagogique "plusieurs groupes cela suppose de réfléchir à une nouvelle distribution des rôles quand on est deux intervenants dans la classe » et parfois même de résister : « moi j'aurais l'impression si j'étais dans ta classe là de rien contrôler, dans la configuration qu'on voit, je me sentirai pas à l'aise et j'ai peur que quand les élèves se retrouvent dans une autre configuration de travail, ils stressent, on devrait penser à tout ça ».

21 Cet extrait illustre la manière dont les enseignants construisent collectivement un point de vue sur un dispositif susceptible selon Juliette de «remotiver les élèves décrocheurs». L'échange professionnel autour des traces vidéo de l'activité de Juliette se construit dans l'expression de points de vue complémentaires sur le travail en îlots mais aussi dans la confrontation des actions et des motifs propres à chaque enseignant. Les discussions induites par les observations sur la séquence filmée se développent autour des mises en œuvre du dispositif allant même jusqu'à interroger les déplacements de Juliette entre les îlots pour évaluer une éventuelle charge de travail supplémentaire. Parfois, des mises en garde sont exprimées par rapport aux enjeux d'apprentissage du travail en îlots remettant en cause les motifs invoqués par Juliette et d'autres collègues. Certaines résistances sont évoquées montrant ainsi que le dispositif présenté peut évoluer et adopter une nouvelle configuration. Le dispositif "îlots", novateur pour certains collègues, est mis en tension avec les formes traditionnelles d'intervention en classe jusque là en vigueur au collège et soulève également des contradictions (envie de contrôler l'organisation en classe /intention de structurer de nouvelles relations professeur-élèves).

22 Les échanges contribuent au développement des points de vue divergents, soulignant en même temps que le dispositif mis en place par Juliette est un "chantier» encore largement à construire. A l'issue de cette étape, la forme que pourrait prendre le travail en "îlots" ainsi que les implications d'une telle organisation au regard des élèves décrocheurs ne sont pas encore stabilisées et nécessitent selon certains enseignants une expérimentation avec leurs élèves.

\subsection{L'expérimentation en classe et l'analyse du potentiel des nouvelles pratiques (étape 4 )}

Lors d'une nouvelle confrontation collective, face à ses collègues, Rose, enseignante de Français, a témoigné de son expérimentation du dispositif « îlots» en classe de $3^{\mathrm{e}} 3$. Le 
premier extrait vidéo montre l'organisation de sa classe, avec des îlots de différentes tailles, constitués de trois à six élèves de même niveau de compétences. Elle explique que l'objectif de sa séance porte sur la préparation de la rédaction avec ses différentes étapes «lecture et analyse du sujet puis construction des différentes parties». Selon cette enseignante, le travail en îlots présente l'intérêt d'apprendre aux élèves à travailler ensemble et de faire rentrer dans le cercle les élèves qui décrochent facilement. L'expression de ces motifs n'évince pas pour autant la difficulté opérationnelle consistant à " passer du mode "classe normale" au mode "îlots" ". Juliette fait part ensuite de sa première analyse "là je vais justement m'appuyer sur le système îlot pour canaliser ces deux élèves [...] ». En se référant aux images vidéo, les collègues soulignent tout d'abord la nécessité de laisser le temps aux élèves de s'approprier une nouvelle configuration de classe «il faut que les élèves comprennent que dorénavant ce sera dans cette architecture que le travail sera organisé » (Pierre); "l'avantage c'est justement que l'élève construise un nouveau mode d'interaction [...] ça prend du temps, là sur ces deux îlots on voit que les élèves interagissent pas mal» (Annie). Certains insistent sur «le cadrage qui doit accompagner un travail en îlot [...] l'îlot est intéressant à partir du moment où tu réussis à amener les élèves à se recentrer sur un travail précis [...] les choses doivent être bien définies, ils doivent savoir ce qu'on attend d'eux dans ce type de travail [...] mais l'îlot ça peut absorber les élèves pas trop motivés ». Les enseignants n'hésitent pas à voir ce nouveau mode de travail en classe comme une occasion de construire de nouvelles interactions émancipatrices mais aussi régulatrices des élèves en décrochage, "absorbés " par la configuration en îlots. Le collectif identifie ainsi le potentiel mais aussi les limites possibles de cette nouvelle forme de pratique « on peut craindre que certains élèves ne maîtrisent pas suffisamment les outils pour interagir en îlots et là on a l'effet inverse ».

$\mathrm{Au}$ cours de cette session de confrontation, nous avons mis en avant les possibles tensions au sein du système d'activité : des tensions en rapport avec le désintérêt des élèves décrocheurs pour une nouvelle organisation de travail et liées à la question de la maîtrise des outils par les élèves (savoir interagir avec ses pairs au sein des îlots); des tensions qui peuvent être amplifiées par la concurrence des motifs de l'enseignant (le souhait d'un autre mode de fonctionnement en classe) et ceux des élèves décrocheurs (le refus de collaborer avec les pairs). L'évocation et la compréhension de ces tensions entre les règles de travail, les outils, le groupe classe, les interactions entre deux systèmes d'activités (enseignant et élèves) ont conduit par la suite le collectif à faire évoluer le dispositif « en îlots » vers trois axes de réflexion : la co-configuration (apprendre aux élèves à interagir entre eux), l'activité saturée (centrer chaque groupe sur un travail précis), la posture en mode central et proximal (développer chez l'enseignant une nouvelle posture démultiplication des déplacements et approche des groupes, pour superviser le travail en îlots). Les tentatives d'adaptation et de re-conception du travail en îlots, aussi formalisé soit-il lors des premières expérimentations, caractérisent cette quatrième étape. Parallèlement à ces formes d'extension et de développement du dispositif en îlots, une nouvelle étape est venue s'ajouter pour répondre aux intentions du collectif. Ainsi, les enseignants ont proposé de recueillir les commentaires des élèves sur les nouvelles modalités de pratique. Mettre à contribution les élèves avec leurs propres points de vue sur le travail en classe (Moussay \& Flavier, 2014) est apparue comme une étape prometteuse au sein du nouvel espace d'analyse du travail. 


\section{Discussion} problématique des décrocheurs d'analyser collectivement les difficultés de mise en activité des élèves et de construire ensemble de nouvelles modalités de pratique. L'intervention a ainsi engagé les enseignants dans une suite d'analyses, elles-mêmes objets d'un nouvel échange sur les manières de faire classe avec les élèves refusant les règles du travail scolaire. Les nouvelles mises en œuvre ont donc été le fruit d'une recherche conjointe de solutions, construites des analyses réalisées in situ menant à la transformation du travail (Kuutti \& Syrjänen, 2011).

Notre intervention a permis aux professionnels volontaires de visionner une série de vidéos sur l'activité, de discuter de modalités d'action concrètes et de partager leurs préoccupations. La mise en visibilité des difficultés voire des impasses face aux élèves décrocheurs, l'identification et l'analyse des tensions propres à l'activité en classe, les savoir-faire et les expériences des pairs sont autant de ressources mises à disposition des enseignants pour prendre le risque d'inventer des nouvelles modalités de pratique et de les mettre à l'épreuve. Ce processus d'inventivité et de créativité nourri par les analyses et les échanges sur l'activité illustre l'orchestration d'une performance collective (Engeström, 2008) distribuée dans le temps (avec une alternance d'observations, d'analyses et d'expérimentations) sur le lieu du travail (établissement scolaire). Les procédures d'échange (à partir de vidéos d'activité), d'expérimentation, de validation ou d'invalidation qui se déploient au sein de ce nouvel espace d'analyse du travail favorisent la démarche collective de transformation des pratiques. L'engagement des professionnels apparait comme le signe d'une revitalisation du collectif de travail (Clot, 2008); une vitalité collective au travail permettant de sortir du décrochage professionnel (Flavier \& Moussay, 2014). La transformation effective des pratiques pédagogiques révèle la capacité du collectif à élargir le rayon d'action (Clot, 2008). Ce qui n'est pas négligeable si l'on envisage le développement du pouvoir d'agir des enseignants comme l'une des conditions pour lutter contre le décrochage des élèves. La perspective d'envisager le processus de décrochage professionnel des enseignants consubstantiel au processus du décrochage scolaire des élèves se voit ici affirmée. En construisant des dispositifs mieux adaptés aux attitudes de refus et de désengagement scolaire, les enseignants réussissent ainsi à renouveler les buts et les motifs, autrement dit le sens de leurs actions auprès des élèves décrocheurs.

Les résultats présentés doivent également être mis en relation avec la question de la collaboration entre chercheurs et professionnels. Comme on a pu le constater à travers notre intervention, cette collaboration qui associe pleinement les enseignants volontaires permet à ces derniers de sortir de l'isolement professionnel et de s'engager dans les transformations de la pratique en affrontant collectivement les problèmes. À visée collaborative, l'intervention orientée vers l'action (Clot, 2008) et le développement professionnel répondent aux besoins des praticiens. Dans l'espace de travail mis en place au sein du collège $\mathrm{T}$, l'objectif est d'articuler une meilleure connaissance et visibilité de l'activité des professionnels confrontés au décrochage avec une perspective d'évolution des pratiques pour une plus grande efficacité des apprentissages. Mais derrière cette expérimentation à l'échelle locale, la collaboration chercheur et professionnels se complexifie avec le partage de la conceptualisation des actions, la coopération au 
processus de développement et en conséquence, renouvelle le cadre épistémologique et éthique d'une telle pratique (Yvon \& Durand, 2012).

\section{Conclusion}

Dans le contexte actuel de réflexion sur les caractéristiques des environnements potentiellement formatifs pour les enseignants, la conception de nouveaux espaces d'analyse du travail au sein des établissements scolaires apparait comme une piste prometteuse de formation (Ria, 2015). Mais cette perspective confirme surtout l'intérêt de mettre à disposition des outils et des méthodes favorisant la reprise de l'expérience du travail, dans le souci d'accompagner les professionnels dans les transformations pédagogiques et de leur permettre d'agir sur les situations de travail (Moussay \& Ria, 2014). Dans les études réalisées au sein des établissements scolaires, l'intervention du chercheur ouvre la voie d'un nouveau questionnement, à savoir celui d'envisager cette intervention comme instrument de la formation d'une nouvelle expérience au service du développement de l'activité par le sens et par l'efficience (Moussay, 2009).

Au-delà de cet enjeu, l'analyse de l'activité en classe autour d'une problématique professionnelle partagée telle que le décrochage, au sein des établissements scolaires par les enseignants de disciplines d'enseignement différentes, ne doit pas s'apparenter à une formation professionnelle ordinaire, dans laquelle l'enseignant formé est souvent passif. Ici les solutions partagées, et discutées ont plus de chances de produire des effets, étant donné que les enseignants sont à la fois acteurs, décideurs, et prescripteurs de leurs choix. De plus, le fait que les solutions envisagées puissent être adoptées par plusieurs acteurs au sein du même établissement, offre la possibilité de concevoir un cadre commun, susceptible, de créer des contextes d'apprentissage plus confortables pour tous. Le partage des analyses sur l'activité professionnelle en groupe interdisciplinaire ouvre le champ de nouvelles pratiques pédagogiques, que nous envisageons à la fois comme des voies d'entrée vers un développement professionnel, au service de la réussite de tous et de chacun des élèves, et comme un levier puissant au service de pratiques pédagogiques novatrices, prenant en compte la nécessité de repenser l'organisation du travail scolaire, dans l'esprit de la nouvelle réforme du collège 2016.

\section{BIBLIOGRAPHIE}

Barrère, A. (2002). Les enseignants au travail. Routines incertaines. Paris : L'Harmattan.

Blanjoie, V. (2014). Analyse de l'activité professionnelle des enseignants d'éducation physique et sportive, résistants à l'usure, en fin de carrière. Mémoire de Master 2, non publié, Université ClermontAuvergne.

Borman, G. D., \& Dowling, N. M. (2008). Teacher attrition and retention: A meta-analytic and narrative review of the research. Review of Educational Research, 78, 367-409.

Clot, Y. (2008). Travail et pouvoir d'agir. Paris : PUF.

Questions Vives, N²5 | 2016 
Clot, Y. (2010). Le travail à cour. Pour en finir avec les risques psychosociaux. Paris: La Découverte.

Clot, Y., \& Kostulski, K. (2011). Intervening for transforming: The horizon of action in the Clinic of Activity. Theory \& Psychology, 21(5) 681-696.

Clot, Y.,\& Gollac, M. (2014). Le travail peut-il devenir supportable? Paris : A. Colin.

Engeström, Y. (2001). Expansive learning at work: toward an activity theoretical reconceptualization. Journal of Education and Work, 14(1), 133-156.

Engeström, Y. (2008). Quand le centre se dérobe : la notion de knotworking et ses promesses. Sociologie du travail, 50, 303-330.

Engeström, Y., \& Sannino, A. (2013). La volition et l'agentivité transformatrice : perspective théorique de l'activité. Revue internationale du CRIRES, vol. 1, 1, 4-19. http://ojs.crires.ulaval.ca/ index.php/ric. Consulté le 13 février 2014.

Flavier, E., \& Moussay, S. (2014). Les réponses des professionnels au décrochage scolaire. Louvain LaNeuve : De Boeck.

Karsenti, T., \& Collin, S. (2009). L'autre décrochage scolaire. Formation et Profession, 16, 2- 6.

Karsenti, T., Collin, S., \& Dumouchel, G. (2013). Le décrochage enseignant : état des connaissances. International Review of Education, 59, 5, 549-568.

Kuutti, K., \& Syrjänen, A-L. (2011). Beyond User Studies and Appropiation: Developmental Work Research. CHI2011 workshop W20: Appropriation and Creative Use: Linking User Studies and Design, Mai 2011, 1-4.

Leontiev, A.N. (1981). Le développement du psychisme. Paris : Editions sociales.

Lothaire S., Dumay, X., \& Dupriez, V. (2012). Pourquoi les enseignants quittent-ils leur école?

Revue française de pédagogie, 181, 99-126.

Macdonald, D. (1999). Teacher attrition : A review of literature. Teaching and Teacher Education, 15, $8,835-848$

Méard, J. (2014). La co-construction de sens dans les interactions entre l'enseignant et les élèves à risque de décrochage. In F. Flavier \& S. Moussay (Eds.). Répondre au décrochage scolaire. Expériences de terrain (pp. 39-50). Bruxelles : De Boeck.

Moussay, S. (2009). Analyse du développement de l'activité professionnelle par le sens et par l'efficience: l'impact de la situation tutorale sur le pouvoir d'agir de trois enseignants débutants. Thèse de doctorat, Université de Montpellier 3, Montpellier, France.

Moussay, S., \& Ria, L. (2014). Nouvelles prescriptions du travail enseignant dans des collèges situés en zone d'éducation prioritaire : quelles transformations de l'activité professionnelle? Revue française de pédagogie, 189, 91-104.

Moussay, S. \& Flavier, E. (2014). L'entretien d'auto-confrontation : la prise en compte du point de vue de l'élève pour développer l'activité en classe. Revue canadienne de l'éducation, 37(1), 96-118.

Mukamurera, J., \& Bouthiette, M. (2008). Rester dans l'enseignement ou quitter ? Portrait de la situation et motivation des enseignants ». Communication séminaire « Pourquoi et comment soutenir l'insertion professionnelle de nouveaux enseignants au Québec ? Résultats de recherche et pistes d'action ». Québec : Université de Sherbrooke.

OCDE (Organisation de coopération et de développement économiques) (2005). Le rôle crucial des enseignants. Attirer, former et retenir des enseignants de qualité. Paris: Editions OCDE. 
Ria, L., \& Moussay, S. (2014). “Change laboratories" within secondary schools: towards accompaniment and support of teachers in the appropriation of new reforms in priority education. In F. Nyhamn \& T. N. Hopfenbeck. From Political Decisions to Change in the Classroom, (pp. 78-95). CIDREE Yearbook.

Ria, L. (2015). Former les enseignants au XXI ${ }^{e}$ siècle - Établissement formateur et vidéoformation. Louvain-la-Neuve : De Boeck - Supérieur.

Sannino, A. (2012). Dialectique et intervention en théorie de l'activité. In Y. Clot (Dir.), Vygotski maintenant, (pp. 213-231). Paris : La Dispute.

Skaalvik, E. M., \& Skaalvik, S. (2011). Teacher Job Satisfaction and Motivation to Leave the Teaching Profession: Relations with School Context, Feeling of Belonging, and Emotional Exhaustion. Teaching and Teacher Education: An International Journal of Research and Studies, 27, 6, 1029-1038.

Virkkunen, J. (2006). Dilemmes dans la construction d'une capacité d'action partagée de transformation. Revue @ctivités, 3(1), 19-42. http://www.activites.org/v3n1/virkkunen-fr.pdf

Virkkunen, J., \& Newnham, D. S. (2013). The Change Laboratory: A tool for collaborative development of work and education. Rotterdam : Sense Publishers.

Vygotski, L. S. (1934/1997). Pensée et langage (3 ed.). Paris : La Dispute.

Yvon, F., \& Durand, M. (2012). Réconcilier recherche et formation par l'analyse de l'activité. Bruxelles : De Boeck.

\section{NOTES}

1. Intervention issue du programme de recherche LéA (lieu d'éducation associé) http://ife.enslyon.fr/lea

2. Enquête par questionnaire sur les besoins en formation (juin, 2014) réalisée par deux enseignantes coordonnatrices du collège

3. Pondération des heures d'enseignement dans les établissements scolaires REP+ et libération de trois demi-journées par année scolaire dans le service des enseignants du second degré

4. Réseau d'éducation prioritaire

\section{RÉSUMÉS}

L'article présente une intervention dont l'objectif est d'aider les enseignants dans la résolution des difficultés professionnelles liées au décrochage scolaire. En référence aux travaux en clinique de l'activité (Clot, 2008), l'intervention développementale consiste à mettre en place, au sein de l'établissement scolaire, un espace d'analyse du travail centré sur le développement de l'activité. Les notes d'observation ainsi que les données des entretiens d'autoconfrontation documentent chacune des étapes conduisant les professionnels à collaborer pour analyser les contradictions et les tensions de leur activité face aux élèves décrocheurs. Les résultats obtenus permettent 
d'envisager l'espace d'analyse du travail implanté dans l'établissement comme un lieu de conception et d'expérimentation de nouvelles mises en œuvre pédagogiques.

The article presents an intervention, which aims to help teachers in solving professional problems related to student dropouts. The developmental intervention is conducted within the framework of the Clinic of Activity studies (Clot, 2008) to establish, within the school, a space of analysis of work centered on the development of professional activity. The observations as well as self-confrontation data document each step leading professionals to analyze together the contradictions and tensions of their activity with dropouts. The results show the space of analysis of work located in the school as a place of conception and experimentation of new pedagogical actions.

\section{INDEX}

Keywords : secondary School, dropout, activity analysis, power to act, collective

Mots-clés : établissement scolaire, décrochage, analyse de l'activité, pouvoir d'agir, collectif

\section{AUTEURS}

SYLVIE MOUSSAY

Université Clermont-Auvergne

\section{VÉRONIQUE BLANJOIE}

Université Clermont-Auvergne 